\title{
Literature Review of Resource Allocation Methods in Cognitive Radio Networks
}

\author{
Zaid Abdul Samad Bardan', S. B. Mule \\ ${ }^{1}$ M.E. Student, SCOE, Pune, India \\ ${ }^{2}$ Assistant Professor, SCOE, Pune, India
}

\begin{abstract}
Cognitive radio network (CRN) is growing worldwide which leads such networks are suffering from the challenges of efficient spectrum/resource allocation as well as lack of spectrum. Efficient spectrum allocation technique becomes new research problem in use of CRN. A major challenge to this new technology is how to make fair assignment of available spectrum to unlicensed users. The appropriate allocation of idle frequency spectrum coexisting cognitive radios while maximizing total bandwidth utilization and minimizing interference is required for the efficient spectrum utilization in CRN. The method of fixed spectrum allocation resulted to less spectrum utilization over the entire spectrum. For cognitive radio systems, Orthogonal Frequency Division Multiplexing (OFDM) widely used data transmission method which delivering the flexibility for allocating the resources under the dynamic conditions. Therefore, OFDM based CRN networks having major challenge of resource allocation. There are different situations and parameters in dynamic environment which are impacting on total system data rate performance. In this paper, we are presenting the survey on cognitive radio networks, spectrum sensing, challenges and different recent methods analysis for resource allocation.
\end{abstract}

Keywords: Cognitive Radio Networks, Resource allocation, Energy

\section{Introduction}

For increasing the employment of the restricted radio information measure currently days the cognitive radio has emerged as a promising technology whereas in wireless networks for services and applications accommodating the increasing quantity. To the dynamic radio environment, a cognitive radio (CR) transceiver is ready to adapt and for the restricted radio resources the network parameters to maximize the employment whereas providing flexibility in wireless access. For the radio environment (in terms of spectrum usage, power spectral density of transmitted /received signals, wireless protocol signaling) and intelligence are the key options of a $\mathrm{Cr}$ transceiver are awareness. For adaptive standardization of system parameters like transmit power, carrier frequency, and modulation strategy (at the physical layer), and higher-layer protocol parameters this intelligence is achieved through learning. Development of cognitive radio technology should upset technical and sensible concerns (which are extremely multidisciplinary) still as regulative needs. There's an increasing interest on this technology among the researchers in each domain and business and therefore the spectrum policy manufacturers. The key enabling techniques for cognitive radio networks (also cited as dynamic spectrum access networks) are broadband signal process techniques for digital radio, advanced wireless communications ways, artificial intelligence and machine learning techniques, and cognitive radio-aware adaptive wireless/mobile networking protocols [7].

To meet user desires from its experiences to reason, plan, and choose future actions A cognitive radio is an adaptive, multi-dimensionally aware, autonomous radio system that learns. For using, permitting access to, or allocating spectrum Standards teams and regulative bodies round the world square measure progressively seeking new ways that. throughout the SDR Forum's world regulative Summit on SDR and cognitive Radio Technologies (June 2005) from round the world mentioned their spectrum management challenges and goals, and therefore the role of recent technologies This was created clear, once standards, regulative and alternative key stake holder representatives. With each the introduction of SDR This interest in developing new spectrum utilization technologies combined and for new and promising technologies like cognitive radio the conclusion that machine learning will be applied to radios is making intriguing prospects. Cognitive Radio and Dynamic Spectrum Access (DSA) represent 2 complementary developments which will produce the globe of wireless communication. A cognitive radio, in contrast, will use data of radio technology and policy, representations of goals, and alternative discourse parameters.

So far regarding cognitive radio and DSA we tend to mentioned, however an ever deficient resource with spectrum turning into, as expeditiously as doable it's fastidious that new systems utilize all accessible frequency bands. Spectrum a lot of dynamically is allocates by Dynamic spectrum access and for analysis it's a vigorous space. Not solely advances in technology however additionally new policy DSA is extremely vital and for spectrum use could be an economic model. Cognitive radios are wide viewed because the riotous technology that may radically ameliorate each spectrum potency and utilization. Cognitive radios are totally programmable wireless devices that may sense their environment and dynamically change their transmission wave form, channel access methodology, spectrum use, and networking protocols as required permanently network and application performance.

For addressing the amount of analysis challenges of mixing the DSA and cognitive radio conferred by analysis community There are totally different analysis works. Between individual analysis results and therefore the largescale preparation of cognitive radio networks there's an enormous gap that dynamically optimize spectrum use. On versatile spectrum use recent developments such LTE-A 


\section{International Journal of Science and Research (IJSR) \\ ISSN (Online): 2319-7064 \\ Index Copernicus Value (2013): 6.14 | Impact Factor (2014): 5.611}

(Long Term Evolution-Advanced) that depends, for cognitive radio and DSA supply colossal opportunities to demonstrate the promising price. For future wireless communications the revolutionary technology bestowed during this survey are at the leading edge. To the present rising technology, outlining the basics of cognitive radio networks and dynamic spectrum access Dynamic spectrum access and cognitive radio networks are equipped an allembracing introduction. during this paper, we tend to are presenting survey study over the cognitive radio networks parts like spectrum sensing, spectrum sharing, spectrum quality, spectrum call etc. additionally totally different spectrum allocation ways mentioned and compared their performances. Section II, presenting literature survey on cognitive radio systems, section III presenting the survey of various spectrum or resource allocation ways for CRN, section IV presenting comparative analysis of various spectrum allocation ways.

\section{Review of Cognitive Radio Components}

\subsection{Cognitive Radio}

Electromagnetic radio spectrum is one in every of the foremost scarce and precious resource. a set spectrum assignment strategy is follow by Wireless networks these days, by government agencies is that the use of that is licenced. as a result of numerous factors like quantity of traffic load on licenced users or geographical variations [1] for allotted spectrum getting used only intermittently or not in any respect This ends up in an outsized portion. for the licenced spectrum by the licenced or primary user (PU) Actual measurements by FCC [2] support this truth by showing a severe underutilization. as a result of restricted accessibility of radio spectrum and high inefficiency in its usage, new insights into the employment of spectrum have challenged the normal approaches to spectrum management. to harness the underutilized wireless spectrum by accessing it opportunistically to harness the underutilized wireless spectrum by accessing it opportunistically, This necessitates a replacement communication paradigm. This new communication technology is referred as Dynamic Spectrum Access (DSA) or cognitive Radio (CR). Derived from J.Mitola's doctoral thesis [4], a cognitive radio is associate degree intelligent wireless communication system that depends on timeserving communication between unauthorized or secondary users (SU) s over briefly unused spectral bands that are licenced to their PUs. The FCC suggests that any radio having reconciling spectrum awareness should be said as - Cognitive Radio [5].

To improve the present spectrum underutilization cognitive Radio systems has been seen as a promising answer whereas the increasing quantity of services and applications in wireless networks [6] is accommodating. With a similar band because the $\mathrm{Pu}$ to at the same time or opportunistically operate cognitive radio technology might probably enable an entire SU system. However, as a result of variety of challenges it faces in however it learns the event of cognitive radio continues to be at a abstract stage and at every finish of the link adapts to the native spectral activity. To acknowledge their communication setting the inherent feature of those metallic element systems would be their ability and for the genus Sus whereas minimizing the interference to maximise the standard of service (QoS) to the PUs adapt the parameters of their communication theme. nevertheless, in channel quality and interference so as to beat high variation $\mathrm{Cr}$ systems have to be compelled to have a high degree of flexibility. In SDR technology as a result of implicit realization of those characteristics it'll be engineered over package outlined radio (SDR), that is already in production and is currently accessible. of the radio setting (in terms of spectrum usage, power spectral density of transmitted/received signals), dynamic ability (adaptive standardization to system parameters like transmit power, carrier frequency, modulation strategy etc.) and extremely economical cooperative or non-cooperative behavior (when there's competition between multiple $\mathrm{Cr}$ transceivers), The key options of $\mathrm{Cr}$ transceivers are awareness. For a $\mathrm{Cr}$ network to be deployed for sensible usage variety of recent technologies have to be compelled to be developed. For specific interest are the challenges concerned within the design of physical and link layers.

For network parameters, reliable spectrum sensing (detecting United Nations used spectrum), spectrum quality (maintaining seamless transition to a replacement spectrum), being (with PUs and different $\mathrm{Cr}$ networks), spectrum management, dependableness (in terms of QoS), resource allocation (such as transmit power allocation and dynamic spectrum sharing (DSS)) so on are variety of recent mechanisms among these layers like measure, have to be compelled to be designed for many economical and much harmless access and sharing of timeserving radio spectrum. additionally, to boost network performance for various things so as it's crucial to best optimize these mechanisms.

For future Cr systems while not inflicting any degradation in commission to PUs, Orthogonal Frequency Division Multiplexing (OFDM) has been known as a possible transmission technology Since plutonium channels have to be compelled to utilize by secondary users in an exceedingly Cr network. In dynamically dynamic spectral environments and allocating unused spectrum among genus Sus this can be principally as a result of its nice flexibility, in radio spectrum to quick dynamic conditions that permits for easy adaptation of sub-carriers. Besides, to boost the spectrum utilization generally OFDM permits for multiuser diversity overcoming frequency selective attenuation that helps. a serious challenge is to design economical resource allocation algorithms (spectrum sharing and power allocation) that works well in OFDM based mostly Cr networks. during this thesis, we tend to specifically check up on these 2 issues of Sub Carrier Allocation, Bit Allocation and power allocation to limb and that we then propose and design sensible algorithms for them.

\subsection{Spectrum Management Functionality}




\section{International Journal of Science and Research (IJSR) \\ ISSN (Online): 2319-7064}

Index Copernicus Value (2013): 6.14 | Impact Factor (2014): 5.611

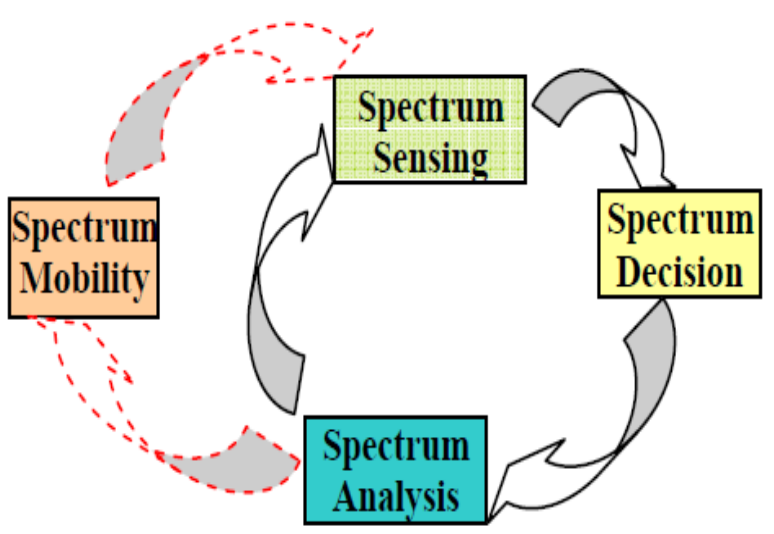

Figure 1: Illustration of spectrum management functionalities

\subsubsection{Spectrum Sensing}

This can be the essential functionality; it consists on sensing unused spectrum and while not interference with the opposite users sharing it. One in every of the goals of the spectrum sensing, particularly for the interference sensing, is to get the spectrum standing (free/busy), so by a SU underneath stress of interference the spectrum may be accessed. By the first transmissions of Sus the challenge is that of activity the interference at the receiver caused.

\subsubsection{Spectrum Decision}

For spectrum access a call model is needed. within the analysis of the spectrum on the parameters thought-about the quality of this model depends. Once a SU has multiple objectives the choice model becomes additional advanced.

\subsection{3. chemical analysis or Sharing}

To estimate the spectrum quality the sensing spectrum results are analysed. One issue here is a way to live the spectrum quality which might be accessed by a SU. By the Signal/Noise Ratio (SNR), the typical correlation and also the accessibility of white areas this quality may be characterised.

\subsubsection{Spectrum quality or Handoff}

To vary its operational frequency Spectrum quality is that the method that enables the Cr user. To control within the best accessible band Cr networks are attempting to use the spectrum dynamically permitting radio terminals, throughout the transition to a more robust frequency to take care of clear communication demand.

\section{Literature Review}

In this section we have a tendency to are discussing totally different strategies planned for dynamic spectrum allocation and resource utilization.

In [5], author presenting approach regarding spectrum convenience in creating the choice during which the cognitive Radio nodes collaborating. To noise ratio conditions with a continuing warning rate at terribly tiny signal Simulation result indicates that each polynomial and linear classifiers offer high detection rate of primary users. As an example, with observation window of fifty bits and 100 percent warning rate the planned strategies can do higher than 90th detection likelihood at $\mathrm{Eb} N o=-7 \mathrm{~dB}$. it's to boot indicated that for each strategies the performance improves as we have a tendency to increase the sensing time.

In [6], author introduced for channel usage time is planned the prediction model, that is predicated on the quality of cognitive Radio users and spectrum transmission spectrum. For the spectrum sharing the channel predicted usage time is main metrics. In sequence from short channel usage time to decrease channel handoffs ensuing, for the channel allocation a usage threshold time is about. Once in an exceedingly single hop channel handoff can not be enforced, to stay the communication the multi-hop routing are going to be established.

In [7], author of this paper for single node spectrum sensing on easy exposure ed and CUSUM based mostly algorithms taking care from our earlier implementation work, in cooperative methodology for ordered modification detection algorithm explained the implementation, the Dual-CUSUM. Single node sensing isn't reliable, notably, due to obstacles (hidden node problem) once the nodes are subjected to shadow weakening. Once mistreatment the expected channel usage time simulation results reveal that the number of channel handoffs is significantly reduced and channel utilization is increased and therefore the handoff block likelihood is additionally dramatically bated at the same time. additionally, the any simulation results show that except the $\mathrm{Pu}$ activity, $\mathrm{Cr}$ node's quality is additionally important to the channel handoffs and link on the market time.

In [9], author of this paper for to handle the difficulty with dynamic channel allocation planned the slotted decision admission management methodology integrated. Within the planned technique, at the start of a brand new slot admitting user solely occurs; therefore, to 1st enter a waiting queue till ensuing slot arrives, new Sus inward between 2 slots ought. By imposing a required to date restricted waiting time on new Sus, to permit admitted Sus to completely utilize the obtainable primary spectrum the planned technique provide a chance. On each the call-level and packet level performances of Sus to analyse the impact of the planned methodology, an analytical framework employing a 3D distinct time markov chain is developed. Simulation result verifies that the accuracy of the analysis and show the effectiveness of the planned methodology in terms of reducing block and dropping possibilities, lowering packet queuing delay, and up spectrum utilization potency.

In [10], in cooperative cognitive networks author propose and analyze the performance of virtual reservation. Through full spectrum utilization to maximise the outturn of the cognitive network virtual reservation could be a narrative link maintenance strategy that aims. Performance analysis shows vital enhancements not only within the Sus block and made termination possibilities however additionally within the outturn of cognitive users.

In [11], in an orthogonal frequency-division multiple-accessbased cognitive radio (CR) network the authors examine resource allocation that dynamically senses primary users (PUs) spectrum and opportunistically uses on the market channels. The aim is resource allocation specified the $\mathrm{Cr}$ 


\section{International Journal of Science and Research (IJSR) \\ ISSN (Online): 2319-7064 \\ Index Copernicus Value (2013): 6.14 | Impact Factor (2014): 5.611}

network outturn is maximised below the PUs most interference constraint and cognitive users (CUs) transmission power budget. Then, to maximise the $\mathrm{Cr}$ network add bit rate (throughput) below the CUs transmission power budget and PUs most interference constraints author formulate the transmission resource allocation downside. During this paper, on a abstract system in such the way that a least square based mostly adaptative algorithm will notice the answer author explained the way to redevelop the subcarrier assignment downside into identical downside outlined.
In [12], for cognitive Radio (CR) systems author investigated the energy consumption issue. For the thought of Cr system with sensible constraints is that the aim of this work is to maximise the energy potency, like the facility budget of the Cr system, the interference thresholds of the first users, the lowest outturn needs and for the Cr users the proportional fairness.

\section{Comparative Analysis}

Below table showing the comparative study among different techniques listed above.

Table 1: Comparative study of resource/spectrum allocation methods

\begin{tabular}{|c|c|c|c|}
\hline $\begin{array}{c}\text { Year of } \\
\text { publication }\end{array}$ & Paper Title & Methodology & Outcomes \\
\hline 2010 & $\begin{array}{c}\text { Comparison of Linear and Polynomial } \\
\text { Classifiers for Co-operative Cognitive } \\
\text { Radio Networks }\end{array}$ & $\begin{array}{l}\text { Cognitive radios, polynomial classifiers; } \\
\text { Linear classifiers; cooperative spectrum } \\
\text { sensing }\end{array}$ & $\begin{array}{c}\text { Detection probability at } \\
\mathrm{Eb} \mathrm{No}=-7 \mathrm{~dB} \text { with observation } \\
\text { window of } 50 \text { bits and } 10 \% \text { false } \\
\text { alarm rate. }\end{array}$ \\
\hline 2011 & $\begin{array}{l}\text { Spectrum Sharing Based on Spectrum } \\
\text { Heterogeneity and Multihop Handoff in } \\
\text { Centralized Cognitive Radio Networks }\end{array}$ & $\begin{array}{c}\text { Cognitive radio; spectrum sharing; } \\
\text { spectrum heterogeneity; multi-hop routing; } \\
\text { spectrum handoff }\end{array}$ & $\begin{array}{c}\text { Improved handoff blocking } \\
\text { probability \& link available time. }\end{array}$ \\
\hline 2011 & $\begin{array}{l}\text { Cooperative spectrum sensing based on } \\
\text { matrix rank minimization }\end{array}$ & $\begin{array}{l}\text { Cooperative sensing, support detection, low } \\
\text { rank property, matrix rank minimization, } \\
\text { cognitive radio }\end{array}$ & $\begin{array}{c}\text { MRM offers large performance gain } \\
\text { over the conventional separate } \\
\text { approach (SA) }\end{array}$ \\
\hline 2012 & $\begin{array}{l}\text { Co-operative Spectrum Sensing: } \\
\text { Implementation and Benchmarking on } \\
\text { ANRC Cognitive Radio Testbed }\end{array}$ & $\begin{array}{c}\text { Cognitive radio(CR), Spectrum Sensing, } \\
\text { Energy detector(ED), GNU Radio, EDD, } \\
\text { real-time emulation, test bed, Sequential } \\
\text { change detection, CUSUM, Dual CUSUM, } \\
\text { Fusion Center (FC) }\end{array}$ & $\begin{array}{c}\text { Dual-CUSUM) is better than its } \\
\text { counterpart Cooperative } \\
\text { Snapshot ED, especially, under low } \\
\text { SNR regimes }\end{array}$ \\
\hline 2013 & $\begin{array}{c}\text { Dynamic Channel Allocation-based Call } \\
\text { Admission Control in Cognitive Radio } \\
\text { Networks }\end{array}$ & $\begin{array}{c}\text { Slotted call admission control method } \\
\text { integrated with dynamic channel allocation }\end{array}$ & $\begin{array}{l}\text { Reducing blocking and dropping } \\
\text { probabilities, lowering packet } \\
\text { queuing delay, and improving } \\
\text { spectrum utilization efficiency }\end{array}$ \\
\hline 2013 & $\begin{array}{c}\text { Improved Spectrum Mobility using } \\
\text { Virtual Reservation in Collaborative } \\
\text { Cognitive Radio Networks }\end{array}$ & $\begin{array}{l}\text { Collaborative Sensing; Link Maintenance; } \\
\text { Reservation; } \\
\text { Admission Control; Real-time systems. }\end{array}$ & $\begin{array}{l}\text { Maximize the throughput of the } \\
\text { cognitive network through full } \\
\text { spectrum } \\
\text { utilization }\end{array}$ \\
\hline 2013 & $\begin{array}{c}\text { Energy-Efficient Resource Allocation in } \\
\text { Cognitive } \\
\text { Radio Systems }\end{array}$ & $\begin{array}{c}\text { A bisection-based algorithm is employed to } \\
\text { work out the optimal solution in an iterative } \\
\text { manner. Developed a fast barrier method to } \\
\text { reduce the computational complexity by } \\
\text { exploiting the problem's structure }\end{array}$ & $\begin{array}{l}\text { Simulation results validate that our } \\
\text { proposed RA scheme can improve } \\
\text { the energy efficiency of } \\
\text { the CR system and the proposed } \\
\text { algorithm converges quickly }\end{array}$ \\
\hline 2015 & $\begin{array}{l}\text { Computationally efficient adaptive } \\
\text { algorithm for resource allocation in } \\
\text { orthogonal frequency-division multiple- } \\
\text { access-based } \\
\text { cognitive radio networks }\end{array}$ & $\begin{array}{l}\text { The problem is formulated as a mixed } \\
\text { integer. Non-linear programming problem } \\
\text { which is NP-hard in general and infeasible } \\
\text { to solve in real-time. }\end{array}$ & $\begin{array}{l}\text { simulation results show that the } \\
\text { achievable bite rates for various } \\
\text { CUs power budget and interference } \\
\text { thresholds are very close to the } \\
\text { maximum achievable bit rates }\end{array}$ \\
\hline
\end{tabular}

\section{Conclusion and Future Work}

This survey paper is aiming at discussing for cognitive radio systems the various aspects, then for spectrum allocation range of recent works, resource allocation, energy potency etc. and at last comparative study among all mentioned works. For cognitive radio networks, in for cognitive networks of achieving the higher performance use of spectrum allocation technique plays important role. For future work, for these existing strategies of cognitive radio networks we advise to figure on mathematical analysis.

\section{References}

[1] Ian F. Akyildiz, Won-Yoel Lee, Mehmat C. Vuran and S. Mohanty, "NeXt gener-ation/dynamic spectrum access/cognitive radio wireless networks: A survey”, in Computer Networks Journal (Elsevier), September 2006

[2] FCC frequency spectrum inventory table:http:/www.fcc.gov/oet/info/database/spectrum

[3] Hisham a Mahmoud, Tevfik Yücek, and Hüseyin Arslan, University of South Florida, "OFDM for Cognitive Radio: Merits and Challenges", IEEE Wireless Communications, April 2009.

[4] J. Mitola III, "Cognitive Radio: An Integrated Agent Architecture for Software Defined Radio", PhD 
Dissertation, Royal Institute of Technology (KTH), Sweden, May 2000.

[5] FCC. Et docket no. 03-322. "Notice of Proposed Rule Making and Order", Dec. 2003.

[6] Z.H. Hashmi, "Dynamic Resource Allocation for Cognitive Radio Systems", Master of Applied Science thesis, The University of British Columbia, June 2008.

[7] Yasmin Hassan and Sharjah, Sharjah, "Comparison of Linear and Polynomial Classifiers for Co-operative Cognitive Radio Networks", 2010.

[8] Guoqin Ning, Jiaqi Duan, Jian Su1, Duo Qiu, "Spectrum Sharing Based on Spectrum Heterogeneity and Multihop Handoff in Centralized Cognitive Radio Networks", 2011.

[9] Ramachandra Budihal and Bharadwaj Desikan, "Cooperative Spectrum Sensing: Implementation and Benchmarking on ANR Cognitive Radio Testbed", 2012.

[10] Shun-Fang Yang and Jung-Shyr Wu1 and Jian-Wei Huang, "Spectrum Handover with Queues and Guard Channels in Cognitive Radio Networks", 2012.

[11]Zhifeng Ni , Hangguan Shan*, Wei Shen t, Jian Wang, "Dynamic Channel Allocation-based Call Admission Control in Cognitive Radio Networks", 2013.

[12] Ayman T. Abdel-Hamid, Ahmed H. Zahran, "Improved Spectrum Mobility using Virtual Reservation in Collaborative Cognitive Radio Networks", 2013.

[13] Mahdi Raeis, Kamal Shahtalebi, Amir Reza Forouzan, "Computationally efficient adaptive algorithm for resource allocation in orthogonal frequency-division multiple-access-based cognitive radio networks", IET Commun., 2015, Vol. 9, Iss. 12, pp. 1442-1449 1442 \& The Institution of Engineering and Technology 2015.

[14]Weijia Shi \& Shaowei Wang, "Energy-Efficient Resource Allocation in Cognitive Radio Systems", 2013 IEEE Wireless Communications and Networking Conference (WCNC): SERVICES \& APPLICATIONS 